\title{
Biorremediação de um Solo Argiloso Contaminado com uma Mistura de Diesel e Biodiesel através da Bioventilação
}

\section{Bioremediation of a Clayey Soil Contaminated with a Mixture of Diesel and Biodiesel by Bioventing}

\author{
Cleomar Reginatto \\ Universidade de Passo Fundo - UPF, Passo Fundo, RS \\ cleomarreginatto@hotmail.com \\ Antonio Thomé \\ Universidade de Passo Fundo - UPF, Passo Fundo, RS \\ thome@upf.br \\ Luciane Maria Colla \\ Universidade de Passo Fundo - UPF, Passo Fundo, RS \\ lmcolla@upf.br \\ Liliane Ribeiro R. Meneghetti \\ Universidade Federal do Rio Grande do Sul - UFRGS, Porto Alegre, RS \\ lilianerebechi@yaboo.com.br \\ Iziquiel Cecchin \\ Universidade de Passo Fundo - UPF, Passo Fundo, RS \\ iziquielc@gmail.com
}

Resumo: O crescente consumo de combustíveis fósseis gera a necessidade de se buscarem fontes alternativas ambientalmente mais adequadas, como é o caso do biodiesel, em misturas com o diesel. Porém essa mistura torna-se um provável contaminante ambiental. A bioventilação é uma técnica de biorremediação que envolve apenas a inserção de ar no meio, para estimular a atividade aeróbia dos microrganismos e surge como uma alternativa aos métodos convencionais de remediação. O objetivo deste

Recebido em 23/02/2012 - Aceito em 15/04/2012.

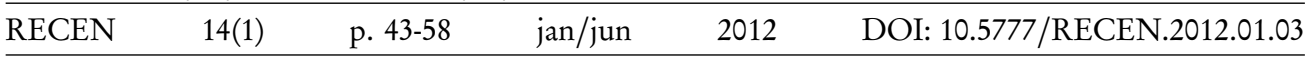


trabalho foi avaliar a biorremediação ex situ de um solo argiloso contaminado com uma mistura B20 utilizando a bioventilação. Os ensaios foram realizados em biorreatores de PVC com 4\% de contaminante, em relação à massa de solo seco. Foram testadas três vazões de ar (2, 4 e $6 \mathrm{~L} / \mathrm{min})$, em três intervalos de tempo distintos (1h a cada $24 \mathrm{~h}, 36 \mathrm{~h}$ e $48 \mathrm{~h}$ ), além do controle. A degradação do contaminante foi avaliada através da determinação da fração residual, extraído por ultrassom, em 3 tempos $(15,30$ e 60 d). A maior degradação aos 60 dias, foi obtida para a velocidade de ar de $6 \mathrm{~L} / \mathrm{min}$ aplicado diariamente, com 86\% de degradação, demonstrando-se ser a bioventilação uma técnica de biorremediação promissora para o tratamento de solos argilosos contaminados com mistura B20.

Palavras-chave: biorremediação; bioventilação; solo contaminado.

\begin{abstract}
The increasing consumption of fossil fuels generates the need to seek more environmentally suitable alternative sources, such as biodiesel in blends with diesel. However the mixture becomes a potential environmental contaminant. Bioventing is a bioremediation technique that involves only the insertion of air in the middle, to stimulate the activity of aerobic microorganisms, and appears as an alternative to conventional methods of remediation. The objective of this study was to evaluate the ex situ bioremediation of a clayey soil contaminated with a mixture B20, using the bioventing. Essays were performed in bioreactors PVC, and $4 \%$ of contaminant on the mass of dry soil. Three air flow rates were tested $(2,4$ and $6 \mathrm{~L} / \mathrm{min})$ at three different time periods ( $1 \mathrm{~h}$ every $24 \mathrm{~h}, 36 \mathrm{~h}$ and $48 \mathrm{~h}$ ), besides of the control. The degradation of the contaminant was evaluated by determining the residual fraction, extracted by ultrasound, in 3 times $(15,30$ and $60 \mathrm{~d})$. The greater degradation at 60 days was obtained for the air speed of $6 \mathrm{~L} / \mathrm{min}$ applied daily with $86 \%$ degradation, demonstrating that the bioventing is a promising bioremediation technique for treating of clay soils contaminated with a mixture B20.
\end{abstract}

Key words: bioremediation; bioventing; contaminated soil. 


\section{Introdução}

O aumento do consumo de combustíveis, as preocupações devido à escassez do petróleo e a necessidade premente de modificação da matriz energética, além dos problemas oriundos das contaminações ambientais por hidrocarbonetos, fizeram surgir os biocombustíveis, como é o caso do biodiesel, que consiste em uma alternativa energética para uso em motores a diesel, pelo fato de ser um combustível renovável [1].

No Brasil, sua aplicação como combustível alternativo como componente junto ao diesel tornou-se obrigatória a partir da lei n ${ }^{\circ}$ 11.097, de 13 de janeiro de 2005 [2]. Em janeiro de 2008, passou a ser obrigatório o uso de $2 \%$ de biodiesel adicionado ao diesel de acordo com as normas da Agência Nacional do Petróleo (ANP) [3]. Em 2009, o percentual foi elevado para 4\% e, em 2010, para 5\%, tendo a previsão de que se possa chegar até a uma mistura B20 (80\% de diesel e 20\% de biodiesel) até 2018.

Nos países da União Européia (UE), especialmente na Alemanha, a mistura atinge $5 \%$ e, às vezes, biodiesel pode ser usado até mesmo em sua forma pura [4].

Essas medidas adotadas diminuem os impactos ambientais negativos decorrentes de um vazamento, considerando-se que, sendo as matérias primas provenientes de origem vegetal ou animal, os contaminantes tornam-se mais biodegradáveis, mas tornam-se também um importante contaminante do solo a ser estudado [3, 5].

Segundo dados da FEPAM [6], entre 2009 e 2011, foram registrados, somente no Rio Grande do Sul, 23 acidentes com cargas envolvendo combustíveis oleosos, principalmente com derrames de diesel e gasolina e destes, três com derrames de biodiesel, sendo o solo o principal meio afetado.

Uma grande variedade de processos físico-químicos e biológicos podem ser utilizados para remediar solos contaminados [7]. Os processos biológicos são uma tecnologia já conhecida internacionalmente e muito desenvolvida, para a remoção de contaminantes oleosos, principalmente devido à eficiência e ao custo quando comparados a alternativas físico-químicas [8, 9].

Os microrganismos são considerados biodegradadores eficientes devido a sua abundância, e diversidade de espécies, e sua versatilidade catabólica e anabólica, bem como a sua capacidade de adaptação a condições ambientais adversas [10]. Os processos de 
biorremediação constituem processos simples e aplicáveis a grandes áreas [11].

Dentre as várias técnicas de biorremediação que podem ser realizadas, as mais utilizadas são a atenuação natural, a bioaumentação e a bioestimulação [11-13].

A técnica de bioventing ou bioventilação como é conhecida, é uma técnica de bioestimulação que se caracteriza pela adição de oxigênio através do solo para estimular o crescimento dos organismos naturais e/ou introduzidos pela bioaumentação.

Quase todos os hidrocarbonetos de petróleo são biodegradáveis sob condições aeróbicas [9]. O oxigênio é um co-substrato que pode iniciar o mecanismo de biodegradação e, depois de iniciado o metabolismo, pode também funcionar como aceptor de elétrons para a geração de energia.

Entretanto, para a aplicação da técnica de bioventilação com eficiência, é necessário o estudo de parâmetros como o tempo de aplicação da técnica e vazão de ar a ser adicionada na área contaminada em solos de granulometria fina, visto estes dados serem deficientes na literatura. Dessa forma, objetivou-se avaliar a influência da vazão de ar e do tempo de ventilação, na biorremediação de um solo argiloso contaminado com uma mistura B20.

\section{Desenvolvimento}

\subsection{Local de estudo}

O estudo foi realizado no Laboratório de Geotecnia Ambiental da Universidade de Passo Fundo, município de Passo Fundo, situado ao norte do estado do Rio Grande do Sul, região Sul do Brasil.

\subsection{Solo}

O solo utilizado é de origem residual e pertence à província geológica do planalto riograndense, e à bacia do Paraná segundo as geologias do Rio Grande do Sul e do Brasil respectivamente [14].

As amostras de solo deformadas foram coletadas do campus experimental de Geotecnia na profundidade de $2 \mathrm{~m}$, no horizonte $\mathrm{B}$, através de uma trincheira aberta no local. 


\begin{tabular}{ll} 
Tabela 1. Caracterização geotécnica e físico-química do solo [25]] \\
\hline Parâmetro & Valor \\
\hline Argila (\%) & 68 \\
Silte (\%) & 5 \\
Areia (\%) & 27 \\
Limite de Liquidez (\%) & 53 \\
Limite de Plasticidade (\%) & 42 \\
Peso específico real dos grãos $\left(\mathrm{kN} / \mathrm{m}^{3}\right)$ & 26,7 \\
Umidade Natural (\%) & 34 \\
Peso específico natural (kN $\left./ \mathrm{m}^{3}\right)$ & 16,3 \\
Índice de Vazios & 1,19 \\
Grau de Saturação (\%) & 75,7 \\
Porosidade (\%) & 54 \\
pH & 5,4 \\
Matéria Orgânica (\%) & $<0,8$ \\
Capacidade de Troca Catiônica $\left(\mathrm{cmolc} / \mathrm{dm}^{3}\right)$ & 8,6 \\
Condutividade hidráulica $(\mathrm{cm} / \mathrm{s})$ & $1,39 \times 10^{-3}$ \\
\hline
\end{tabular}

A tabela 1 apresenta algumas características geotécnicas e físico-químicas do solo, a partir dos dados de outro estudo realizado com o solo do mesmo local deste estudo. Já na figura 1, apresenta-se a curva característica do solo.

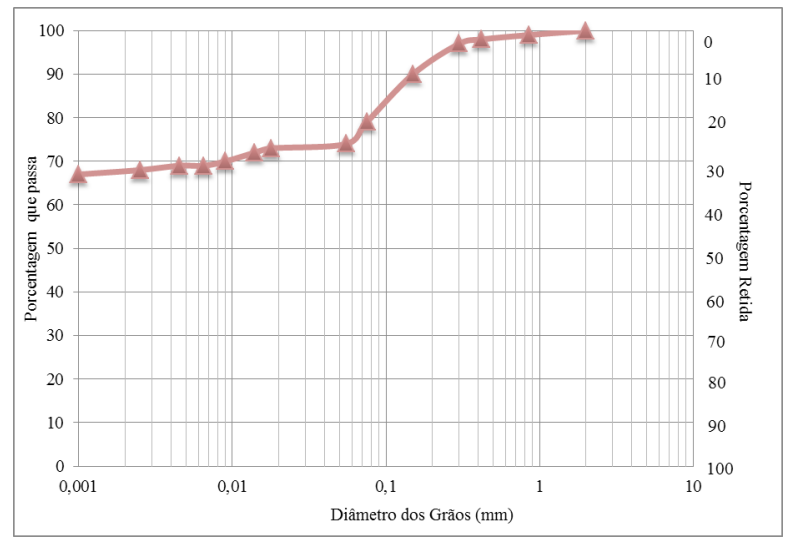

Figura 1. Curva característica do solo de estudo

A classificação pedológica é de um Latossolo Vermelho Distrófico Húmico (unidade Passo Fundo) [15]. Do ponto de vista geotécnico, é classificado como $\mathrm{CH}$, ou 
argila de alta plasticidade.

O solo apresenta $\mathrm{pH}$ ácido, alto teor de argila, baixo teor de matéria orgânica e baixa CTC, típica de solos com predominância do argilo-mineral caulinita [15].

\subsection{Moldagem dos corpos de prova (CP)}

O solo foi peneirado com peneira ASTM n ${ }^{\circ} 10$ e ajustada a umidade para $30 \%$. Posteriormente, as amostras foram contaminadas e homogeneizadas individualmente para cada corpo de prova.

O solo foi contaminado com 4,0\% de B20 sobre o peso seco de solo, o equivalente a uma contaminação de $40 \mathrm{~g} / \mathrm{kg}$ de solo. Os CPs foram moldados em uma prensa hidráulica, na massa específica natural $\left(1,6 \mathrm{~g} / \mathrm{cm}^{3}\right)$, com diâmetro de $6,6 \mathrm{~cm}$ e altura de $10 \mathrm{~cm}$, sendo que a massa de cada CP foi de $0,547 \mathrm{~kg}$ e volume de $342 \mathrm{~cm}^{3}$.

\subsection{Bioventilação}

O solo foi bioestimulado por meio da utilização de ar comprimido. A bioventilação foi realizada com o auxílio de um compressor, através de um fluxo ascendente de ar. Foram testadas três diferentes vazões (2, 4 e 6 L/min; V1, V2 e V3 respectivamente) e três diferentes intervalos de tempo, de $1 \mathrm{~h}$ a cada $24 \mathrm{~h}, 1 \mathrm{~h}$ a cada $36 \mathrm{~h}$ e $1 \mathrm{~h}$ a cada 48 h, $6 \mathrm{~d} /$ semana.

\subsection{Montagem dos biorreatores}

Os biorreatores foram construídos com tubos de PVC, com 100 mm de diâmetro e $200 \mathrm{~mm}$ de comprimento. Na extremidade inferior (cap), uma abertura com válvula permitiu a entrada do ar comprimido para bioventilação e, na parte superior, foi colocado outro cap apenas para evitar a entrada de quaisquer matérias que pudessem interferir nas análises, porém este não impedia a passagem do ar. A figura 2 apresenta um esquema dos biorreatores.

Os CPs foram fixados no interior dos tubos de PVC com gesso, permanecendo assim em estado confinado. Ainda o cap inferior do tubo foi fixado com silicone no tubo para forçar o ar a passar pelo corpo de prova. Na figura 3 , é apresentado um 
esquema completo do sistema de bioventilação.

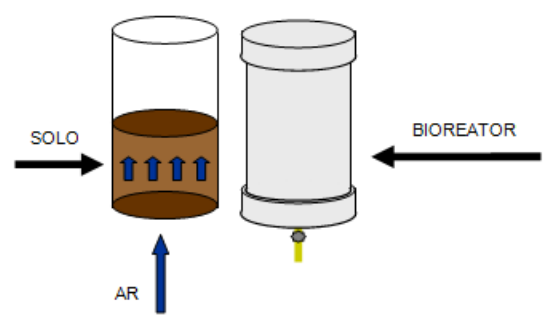

Figura 2. Biorreatores com passagem de ar ascendente

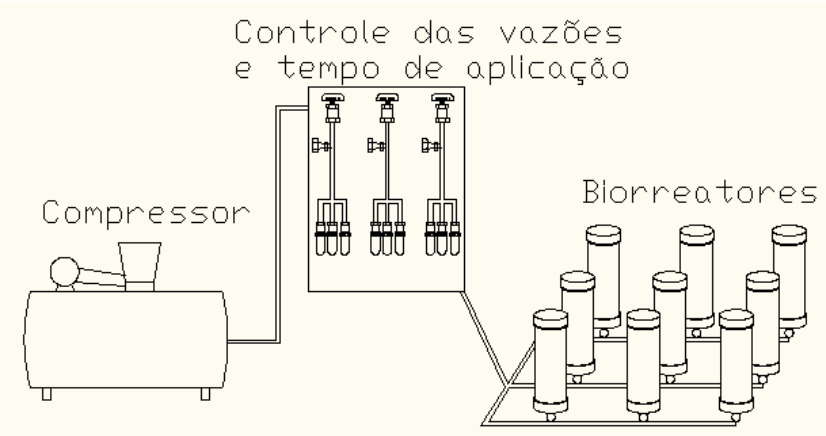

Figura 3. Biorreatores utilizados no experimento

O experimento foi conduzido em laboratório com temperatura controlada de $24^{\circ} \mathrm{C}$, seguindo um delineamento fatorial completo $3^{2}$, sendo as variáveis independentes: a vazão e o tempo de aplicação, cada uma variando em três níveis, totalizando 9 ensaios. Um controle foi realizado sem a adição da bioventilação, através do tratamento do solo por atenuação natural (Tabela 2). Réplicas dos ensaios foram realizadas nos pontos centrais das variáveis para a estimativa da variabilidade dos ensaios.

Em cada tempo de coleta foi retirado um biorreator para cada amostra.

\subsection{Avaliação da biorremediação}

Foram feitas análises da degradação do contaminante em intervalos de 15, $30 \mathrm{e}$ 60 dias após o início do experimento. Para avaliação da biodegradação os corpos, de prova foram desestruturados e o solo utilizado para a análise quantitativa da degradação do B20, através da extração de óleos e graxos residual utilizando ultrasom por sonda, seguindo a metodologia da USEPA 3550B [16]. 
Revista Ciências Exatas e Naturais, Vol.14, n 1, Jan/Jun 2012

Foi utilizado um desruptor de células da marca Unique, modelo De S500, empregando a macro ponta com potência de $99 \%$ e tempo de extração de 2 min.

\subsection{Tratamento dos dados e análise estatística}

Os resultados foram avaliados pela Análise de Variância e comparação de médias pelo Teste de Tukey a $5 \%$ de significância, utilizando o software Statistica $5.5{ }^{\circledR}$.

\section{Resultados}

$\mathrm{Na}$ tabela 2 estão apresentados os teores residuais do contaminante para cada tempo e vazão de ar utilizada. Verifica-se que os resultados de teores de óleos e graxas apresentam valores mais uniformes nos primeiros 15 dias, passando a valores residuais mais diferenciados pela vazão e tempo de aplicação de ar após os 30 dias de ensaio.

Tabela 2. Percentual de óleos e graxas no solo durante o tempo de biorremediação

\begin{tabular}{lllll}
\hline Amostra & \multicolumn{4}{c}{ Tempo de Análise (d) } \\
\cline { 2 - 5 } & 0 & 15 & 30 & 60 \\
\hline V1 - 1/24 & 4 & 1,84 & 1,58 & 0,82 \\
V1 - 1/36 & 4 & 1,88 & 1,36 & 0,72 \\
V1 - 1/48 & 4 & 1,76 & 1,63 & 0,90 \\
V2 - 1/24 & 4 & 1,67 & 1,37 & 0,79 \\
V2 - 1/36 & 4 & 2,04 & 1,42 & 0,82 \\
V2 - 1/48 & 4 & 1,58 & 1,18 & 0,88 \\
V3 - 1/24 & 4 & 1,99 & 0,93 & 0,54 \\
V3 - 1/36 & 4 & 1,96 & 0,91 & 0,59 \\
V3 - 1/48 & 4 & 1,67 & 1,15 & 0,62 \\
At. Natural & 4 & 2,2 & 1,72 & 1,45 \\
\hline
\end{tabular}

As amostras V1, V2 e V3 representam, respectivamente, as vazões de 2,4 e 6 $\mathrm{L} / \mathrm{min}$, sendo que se observa que, após $60 \mathrm{~d}$ de ensaio, a maior vazão (V3) foi a que apresentou os menores valores de percentual de óleos e graxas. Nas figuras 4, 5 e 6 estão apresentados os valores da fração residual em cada tempo de análise para cada uma das três vazões de bioventilação, comparando com o valor de atenuação natural. 
REGINATTO, C. et al

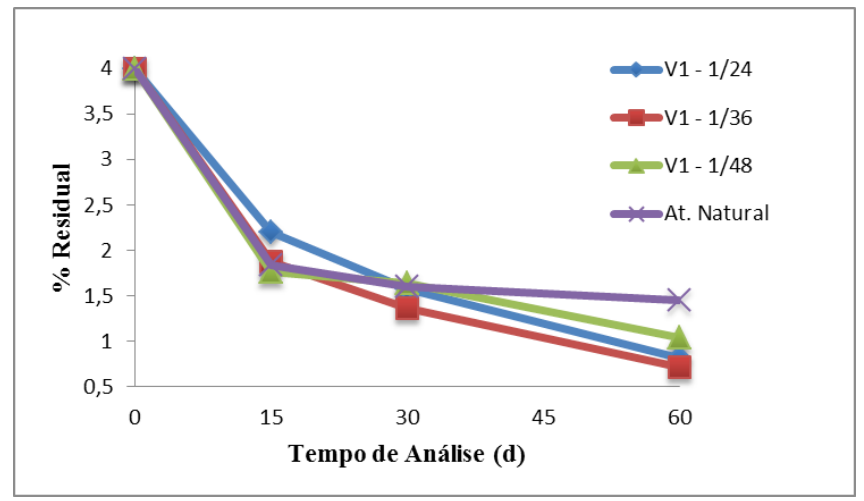

Figura 4. Fração residual dos contaminates com a V1 comparado com a atenuação natural

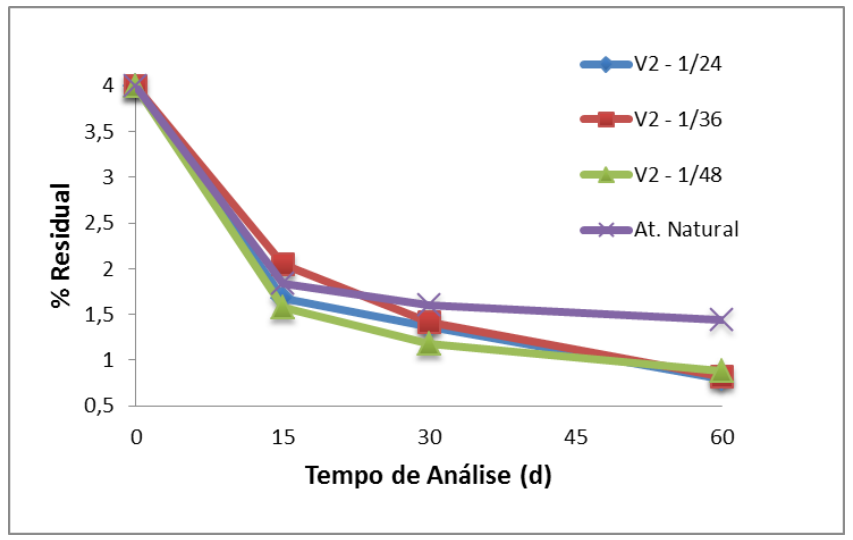

Figura 5. Fração residual dos contaminates com a V2 comparado com a atenuação natural

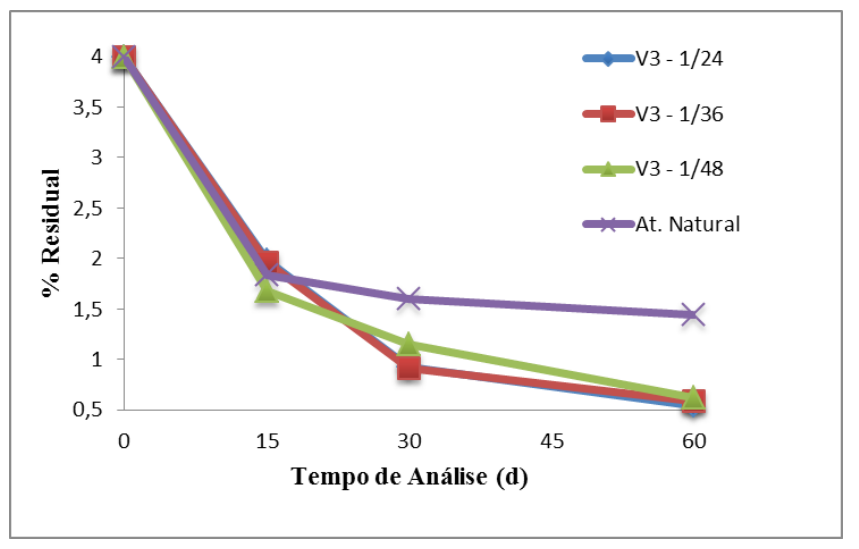

Figura 6. Fração residual dos contaminates com a V3 comparado com a atenuação natural 
Verifica-se que os ensaios bioventilados apresentaram menores valores de percentual de óleos e graxas ao longo do tempo em comparação com o ensaio de atenuação natural.

O processo natural de um poluente orgânico no solo, sem a adição de nutrientes ou qualquer adequação das condições ambientais, pode ocorrer lentamente devido à adaptação dos microrganismos do solo natural [17].

O percentual de degradação para cada uma das amostras nos diferentes tempos de análise está apresentado na tabela 3 .

Tabela 3. Percentual de degradação de resíduos oleosos nas amostras

\begin{tabular}{llll}
\hline Amostra & \multicolumn{3}{l}{ Tempo de biorremediação (d) } \\
\cline { 2 - 4 } & 15 & 30 & 60 \\
\hline V1-1/24 & $53,99 \mathrm{~A}$ & $60,56 \mathrm{AB}$ & 79,62 A \\
V1-1/36 & $53,08 \mathrm{~A}$ & $66,02 \mathrm{AB}$ & $82,02 \mathrm{~A}$ \\
V1-1/48 & $55,98 \mathrm{~A}$ & $59,15 \mathrm{AB}$ & $77,4 \mathrm{~A}$ \\
V2-1/24 & $58,15 \mathrm{~A}$ & $65,84 \mathrm{AB}$ & $80,14 \mathrm{~A}$ \\
V2-1/36 & $50,36 \mathrm{~A}$ & $66,6 \mathrm{AB}$ & $79,51 \mathrm{~A}$ \\
V2-1/48 & $60,51 \mathrm{~A}$ & $70,6 \mathrm{AB}$ & $78,08 \mathrm{~A}$ \\
V3-1/24 & $50,36 \mathrm{~A}$ & $76,76 \mathrm{~A}$ & $86,47 \mathrm{~A}$ \\
V3-1/36 & $50,91 \mathrm{~A}$ & $77,29 \mathrm{~A}$ & $85,27 \mathrm{~A}$ \\
V3-1/48 & $58,15 \mathrm{~A}$ & $71,25 \mathrm{AB}$ & $84,59 \mathrm{~A}$ \\
At. Natural & $44,93 \mathrm{~A}$ & $57,04 \mathrm{~B}$ & $63,86 \mathrm{~B}$ \\
\hline
\end{tabular}

*Letras iguais entre as mesmas colunas, não apresentam diferenças estatísticas entre si

Pela tabela 3 , observa-se que a velocidade de $6 \mathrm{~L} / \mathrm{min}$ (V3) apresentou maior percentual de degradação ao final de 60 d, em relação a todas as demais amostras. Essa degradação foi maior nos três tempos aplicação de ar 1/24, 1/36 e 1/48h.

Após 60 dias de ensaio, obteve-se uma degradação de 86,4\% para a amostra V3$1 / 24$, seguida de uma maior degradação das amostras V3-1/36 e V3-1/48 com 85\% e $84 \%$ de degradação respectivamente, sendo que a atenuação natural apresentou degradação de $63 \%$.

Observa-se, também que, pelos dados de $60 \mathrm{~d}$, tanto as amostras com a velocidade de $4 \mathrm{~L} / \mathrm{min}$ como de $6 \mathrm{~L} / \mathrm{min}$ apresentaram degradação maior quanto menor o tempo entre as aplicações de ar. Dados de outros autores, as quais testaram diferentes 
tempos de aplicação de ar, em que concluiram que um menor tempo entre uma aplicação e outra aumentou a degradação dos contaminantes, corroboram os resultados apresentados [18].

A tecnologia de bioventilação é um importante meio de remoção, através de volatilização, da massa do contaminante e sua destruição in situ. A aplicação de ar e/ou oxigênio puro na camada de subsuperfície, estimula o crescimento da população existente, resultando na redução, via potencial oxidativo, da microbiota dos contaminantes do local [19].

A análise de variância das degradações, mostrada na tabela 3 , apresentou diferenças significativas entre o intervalo de tempo entre as aplicações e a vazão de ar com um nível de significância de 0,0068 (considerando a vazão e intervalo de tempo de 0 a atenuação natural).

Após 15 dias de ensaio, não se observaram diferenças significativas entre as amostras. No tempo de 30 dias, observaram-se diferenças significativas entre as amostras V3-1/24 e V3-1/36 com a atenuação natural, todas as demais amostras não apresentam diferenças significativas entre si.

Já no tempo de 60 dias se observa diferença significativa apenas para a vazão e intervalo de tempo entre as aplicações 0 (atenuação natural), comparada com as demais amostras. Diferentes intervalos de tempo entre as aplicações de ar e as outras 3 vazões não apresentaram diferenças significativas para nenhum dos tempos analisados.

As vazões baixas de ar são suficientes para bioestimular os microrganismos do solo, resultando em uma eficiente degradação [20]. Outros autores também estudaram diferentes tempos entre as aplicações de ar, e os resultados mostraram que, para vazões próximas, não se teve diferenças significativas entre os resultados, mas que a técnica de bioventilação apresentou bom resultado de degradação [18].

Os bons resultados de degradação apresentam influência pela presença do biodiesel nas amostras. Inicialmente os microrganismos utilizaram fontes de carbono mais lábeis como a fração biodiesel e hidrocarbonetos alifáticos, assim provavelmente conseguiram estabelecer uma estrutura populacional significativa após um período de adaptação e também puderam exibir um suporte enzimático, a fim de biodisponibilizar uma quantidade significativa de carbono de fácil obtenção para produção de 
energia pela célula - o que possivelmente contribuiu para a degradação das frações mais recalcitrantes da mistura, como os hidrocarbonetos aromáticos policíclicos. Vários autores reforçam que a biodegradação das frações pesadas de derivados do petróleo pode ser tanto estimulada quanto aumentada, devido ao fenômeno do cometabolismo microbiano [21,22]. Esse fenômeno vem sendo bastante discutido e estudado, principalmente para misturas de diesel/biodiesel [21, 23].

A diferença significativa de degradação entre a bioventilação e a atenuação natural se deve ao fato que, como se trata de amostras de solo sem histórico de contaminação, os microrganismos autóctones foram submetidos a um intenso processo de adaptação e, provavelmente, uma intensa reorganização da estrutura e composição da população microbiana, em virtude do contaminante. Isto também ocorre nos experimentos de outro autor, para um solo argiloso [24]. Tempos maiores de análise possivelmente resultarão em melhores resultados para a bioventilação.

\section{Conclusões}

A técnica de bioventilação apresentou maior eficiência quando comparada a técnica de atenuação natural em todas as combinações de velocidade de ar e intervalo de tempo entre as aplicações, porém apenas no tempo final se observaram diferenças significativas entre a bioventilação e a atenuação natural.

Não se observaram diferenças significativas entre as três vazões e intervlos de tempo entre as aplicações de ar.

Os melhores resultados de degradação chegaram a 86\% para a amostra V3-1/24, sendo que a atenuação natural chegou a valores de $63 \%$.

A técnica de bioventilação é uma boa técnica para ser utilizada para biorremediar solos argilosos contaminados por diesel e misturas.

\section{Referências}

[1] DEMIRBAS, A. Importance of biodiesel as transportation fuel. Energ Policy, v. 35, p. 4661-4670, 2007.

[2] BRASIL. Decreto-Lei $n^{\circ}$ 11.097, de 13 de janeiro de 2005. Dispõe so- 
bre a introdução do biodiesel na matriz energética brasileira, altera as leis $n^{\circ}$ 9.478, de 6 de agosto de 1997, 9.847, de 26 de outubro de 1999, e 10.636, de 30 de dezembro de 2002, e dá outras providencias. Diário Oficial, Brasília. Seção 1, v. 142, n. 10, p. 8, 2005. Disponível em: http://nxt.anp.gov.br/NXT/gateway.dllf=templates\&fn=default.htm\&vid=an p:10.1048/enu. Acesso em dez/2010.

[3] SOARES JR, J.; MARIANO, A.P; DE ANGELIS, D. F. Biodegradation of biodiesel/diesel blends by Candida viswanathii. Afr J Biotechnol, v. 8, n.12, p. 2774-2778, 2009.

[4] SCHLEICHER, T. R.; WERKMEISTER, W.; MEYER-PITTROFF, R. R. Microbiological stability of biodiesel-diesel-mixtures. Bioresource Technol, v. 100, p. 724-730, 2009.

[5] CHIARANDA, H. S.; SCHAMBECK, C.M.; CORSEUIL, H.X. Avaliação da atenuação natural do biodiesel de soja puro (B100) e da mistura diesel/biodiesel (B20) em sistemas subsuperficiais. In: Anais $25^{\circ}$ Congresso Brasileiro de Engenharia Sanitária e Ambiental. Recife. 2009.

[6] FEPAM-RS - Fundação Estadual de Proteção Ambiental Henrique Luiz Roessler. Acidentes de transporte de cargas perigosas. Disponível em: http://www.fepam.rs.gov.br/emergencia/rel_acidentes.asppagina_Atual=28na vegacao $=$ next\&ordem. Acesso em jan/2012.

[7] FAISAL I. K.; HUSAIN, T.; HEJAZI, R. An overview and analysis of site remediation technologies. J Environ Manage, v. 71, p. 95-122, 2004.

[8] ABDULSALAM, S; OMALE, A. B. Comparison of biostimulation and bioaugmentation techniques for the remediation of used motor oil contaminated soil. Braz Arch Biol Techn, v. 52, p. 747-754, 2009.

[9] KARAMALIDIS, A. K.; EVANGELOU A. C.; KARABIKA E.; KOUKKOU,A. I.; DRAINAS, C.; VOUDRIAS, E. A. Laboratory scale bioremediation 
of petroleum contaminated soil by indigenous microorganisms and added Pseudomonas aeruginosa strain Spet. Bioresource Technol, v. 101, p. 6545-6552, 2010.

[10] MORAES, B. E.; TORNISIELO, T. M. S. Biodegradation of oil refinery residues using mixed- culture of microorganisms isolated from a landfarming. Braz Arch Biol Techn, v. 52, p. 1571-1578, 2009.

[11] BENTO, F. M.; CAMARGO, F. A. O.; OKEKE, B. Bioremediation of soil contaminated by diesel oil. Braz J Microbiol, v. 34, p. 65-68, 2004.

[12] LIN T.; PO-TSEN P.; SHENG-SHUNG C. Ex situ bioremediation of oilcontaminated soil. J Hazard Mater, v. 176, p. 27-34, 2010.

[13] MARTINS, A. P. R.; DINARDI, A. L.; FORMAGI, V. M.; OPES, T. A.; BARROS, R.; CONEGLIAN, C. M. R; BRITO, N . N.;DRAGONI, S. G.; TONSO, S. Biorremediação. In: III Fórum de Estudos Contábeis, 2003, Rio Claro. III Fórum de Estudos Contábeis, São Paulo, Brasil, 2003.

[14] BERTORELLI, A. E HARALYI, N. Geologia do Brasil. In: Oliveira, A. M. S.; Brito, S. N. A. (Coord.) Geologia de engenharia. São Paulo: Associação Brasileira de Geologia de Engenharia. 1998, p. 584.

[15] STRECK, E. V. Solos do Rio Grande do Sul. $2^{a}$ ed., Porto Alegre: EMATER/RS, Brasil, 2008.

[16] USEPA (United States Environmental Protection Agency). Method 3550B, ultrasonic extraction, (1996). Disponível em http://www.epa.gov/sw846/pdfs/3550b.pdf. Acesso em fev/2011.

[17] BAPTISTA, P. M.; RIZZO, A. C. L. Acompanhamento do processo de atenuação natural de solo contaminado por petróleo. In: XII Jornada de Iniciação Científica do CETEM/MCT. 2004.

[18] EUI-YOUNG, H.; PARK, J. , KIM, J.; NAMKOONG, W. Effects of aeration mode on the composting of diesel-contaminated soil. J Ind Eng Chem, v. 12, n. 5, p. 694-701, 2006. 
[19] FERNANDES, F. M.; ALCÂNTARA, G. Z. Biorremediação de solos - Estado da Arte.Disponível em: http://pt.scribd.com/doc/56616692/Contaminacao-desolo-bioventilacao. Acesso em jan/2012.

[20] IM-GYU, B.; NAM, H.; SONG, S. K.; HWANG, I.; LEE, T.; PARK, T. Monitoring of bioventing process for diesel-contaminated soil by dehydrogenase activity, microbial counts and the ratio of n-Alkane/Isoprenoid. Korean J. Chem Eng, v. 22, p. 917-921, 2005.

[21] PASQUALINO, J. C., MONTANE, D.; SALVADO, J. Synergic effects of biodiesel in the biodegradability of fossil-derived fuels. Biomass Bioenerg, v. 30, p. 874-879, 2006.

[22] ZHANG X.; PETERSON C. L.; REECE D.; HAWS R.; MOLLER G. Biodegradability of biodiesel in the aquatic environment. Trans ASAE, v. 41, p. 1423-1430, 1998.

[23] OWSIANIAK, M.; LUKASZ C. A. S.; STANIEWSKI J.;ANDRZEJ O.; AGNIESZKA K.; OLEJNIK S.; HERMANN J. Biodegradation of diesel/biodiesel blends by a consortium of hydrocarbon degraders: Effect of the type of blend and the addition of biosurfactants. Bioresource Technol, v. 100, p. 1497-1500, 2009.

[24] MEYER, D. D. Avaliação da biodegradabilidade de misturas de diesel e biodiesel (B0, B20 e B100) em dois solos com diferentes granulometrias. Dissertação de Mestrado. Universidade Federal do Rio Grande do Sul, Porto Alegre, 2011.

[25] PRIETTO, P. D.; REGINATTO, C.; KORF, E. P.; THOMÉ, A.;CANSOLI, N.C. Comportamento hidráulico e reativo de uma mistura solo-cimento para barreiras de fundo em aterros de resíduos sólidos. In: Congresso Brasileiro de Mecânica dos Solos e Engenharia Geotécnica. $15^{a}$ ed., ABMS, Gramado, RS, Brasil. CD-ROM, 2010.

[26] SPINELLI, L. F. Biorremediação, toxicidade e lesão celular em derrames de gasolina. Tese de Doutorado, Faculdade de Engenharia Civil, Universidade Federal do Rio Grande do Sul, Porto Alegre, Brasil, 2005. 